\title{
Paper Relay-assisted WDM-FSO System: A Better Solution for Communication under Rain and Haze Weather Conditions
}

\author{
Navneet Dayal, Preeti Singh, and Pardeep Kaur \\ Department of Electronics \& Communication Engineering, UIET, Chandigarh, India
}

https://doi.org/10.26636/jtit.2017.113917

\begin{abstract}
Among various conventional wireless communication systems, Free Space Optics (FSO) may be distinguished as well, which provides a good level security, high data rates, an enormous bandwidth and negligible susceptibility to electromagnetic interference. The main problem arises with unfavorable weather conditions, which affect the system's parameters and require an aversion to other paths. The weather conditions discussed are rain and haze. Elimination of their adverse effects is essential for establishing a better communication system. The routing path is a major issue, as its decreased parameters lead to the diversion of the transmitted signal or to its total loss. In this article, increased system path length and proficiency levels are demonstrated by using relays, which are assisted by Wavelength Division Multiplexing (WDM).
\end{abstract}

Keywords-Free Space Optics, Wavelength Division Multiplexing.

\section{Introduction}

The boost in the communication field is observed in the $700-1800 \mathrm{~nm}$ range of the spectrum. These high frequency waves are not only untraceable due to their narrow beam footprint, but also offer sufficient bandwidth. The basic communication system consists of a transmitter (LED or laser), a communication medium (air or vacuum), and a receiver which can be a photodiode or a PIN diode. The necessary condition for the Free Space Optics (FSO) system to work is Line of Sight (LOS) between transceivers [1]-[3]. If LOS is available, FSO outperforms all other systems by offering numerous benefits, including a lower bit error rate and lower cost, a higher signal-to-noise ratio, greater security excessive bandwidth, a sufficient data rate of up to tens of $\mathrm{Gb} / \mathrm{s}$, no interference with electromagnetic waves, a minimum Doppler spread etc. The maximum information capacity of any communication system is given by the Shannon Hartley's theorem.

$$
C=B \log _{2}\left(1+\frac{S}{N}\right) .
$$

It presents the relation between bandwidth $B$ (in $\mathrm{Hz}$ ) and information capacity $C$ in bits, where $S$ is the average signal power, with $N$ being the average noise power.
One can notice that capacity is directly dependent on bandwidth. Thus, it is correct to say that FSO operates as the first and last mile access gateway to communication networks. Having such huge advantages, FSO has made its way to all telecom-related fields and is used in a multitude of applications, such as disaster recovery, when all the connections are disrupted, as well as various defense operations, aircraft links, ground-to-airplane links, backhaul connectivity, and enterprise links. All of these applications rely on FSO. In spite of offering all well-known benefits and efficiency-related advantages, FSO is still suffering from a lot of drawbacks [4]. Due to turbulence resulting from a varying refractive index present in the different layers of the atmosphere, there is a loss of signal strength during propagation. These changes depend on the behavior of atmospheric molecules during any given weather situation. Therefore, as weather conditions change, the effect on signal changes accordingly.

Signal attenuation during heavy rain, haze, fog, storm, etc. is high compared to other conditions, so efforts should focus on making the system better. Such conditions cause laser light to either deviate or reflect, depending on the type of scattering effect offered by the molecules present and the wavelength transmitted. The loss that occurs in the path of the communication medium is due to many factors, such as scattering, absorption, pointing errors, scintillation caused by temperature and pressure changes resulting from the packet heating the air molecules. $1550 \mathrm{~nm}$ is referred to as the minimum absorption window. Performance of the system is judged using the Bit Error Rate

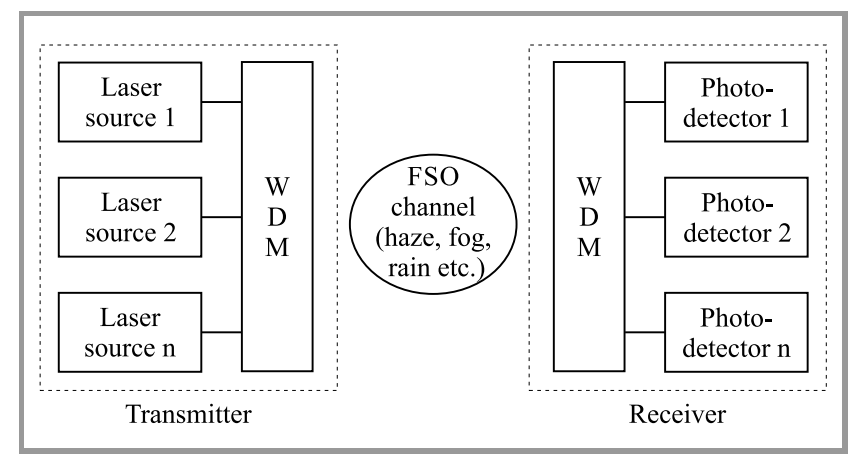

Fig. 1. Example of WDM-FSO system. 


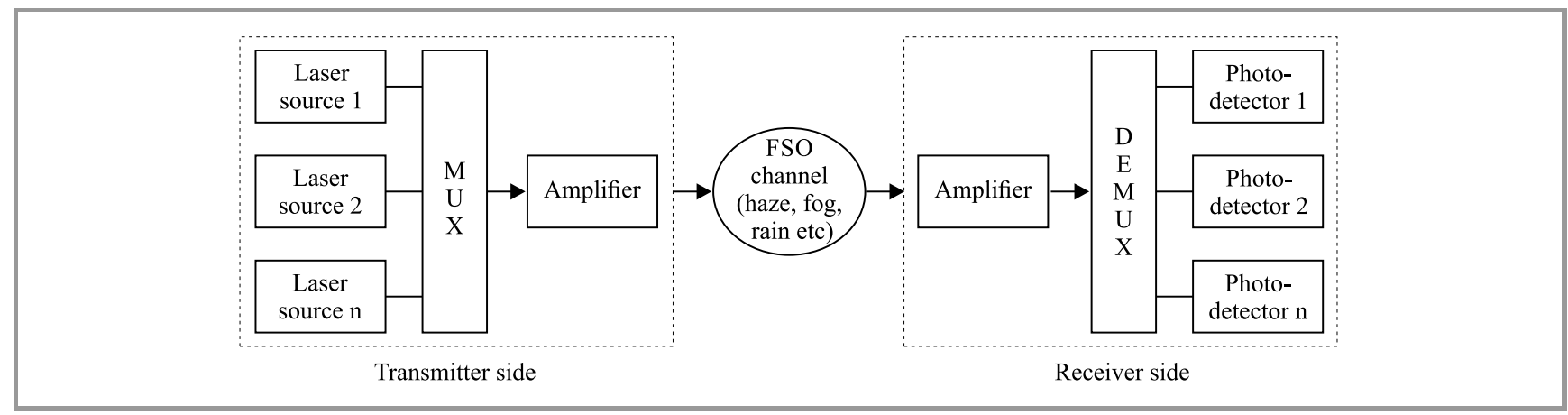

Fig. 2. An amplifier-assisted network shown along with the WDM system.

(BER) and the Q factor which depends on the transmitted power of the laser. The attenuation of light, as per the Beer-Lambert law, is:

$$
T(R)=\frac{P(D)}{P(S)}=\mathrm{e}^{-A R}
$$

where $T(R)$ is the transmittance at a distance $R, A$ is atmospheric attenuation, $P(D)$ is the power received at the receiver and $P(S)$ is the power at the transmitter.

WDM is a technique (Fig. 1) which is used to enhance the system's capacity, as number of wavelengths are accommodated within a single channel [5]-[6]. But due to the threat of non-linearity it possesses, the use of WDM systems requires different components, such as EDFA amplifiers, to ensure a good transmission range and SNR. EDFAs boost the signal and thus increase the SNR at the output [7]. The typical configuration is shown in Fig. 2. It describes the use of optical amplifiers at two positions, rather than using one for each user before WDM, thus decreasing the number of devices and ensuring good range and quality.

A new concept of relays has now been introduced to WDMFSO. This combination is a good step in the direction to achieve optimum communication. The transmitter and the receiver act as the source and destination of the FSO network, while the air is the medium. It is there where relays are incorporated, as shown in Fig. 3. A relay is different than an optocoupler and behaves as a regenerator in the receiving circuit. Those devices which are activated by the incoming signal and are used to connect or disconnect a part of the circuit within the system.

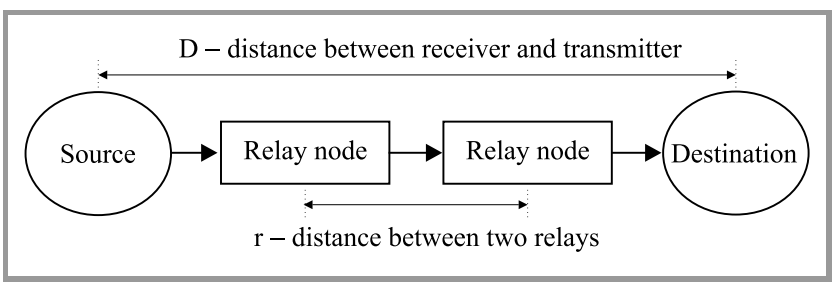

Fig. 3. Relay-assisted network.

Relays ensure complete isolation of the system from the external noise, increasing operation speed [8]-[9]. In optics, this isolation is supplied with a modulated laser. Relays al- low to setup a virtual system between the transmitter and the receiver, so that the signal is received even if there is no LOS. Thus, it is referred to as a cooperative diversity system. Such a concept was first proposed by Acompora and Krishnamurthy in 1999, showing that the area coverage can be increased and outage probability can be minimized.

\section{System Analysis}

Rain is one of the most degrading factors disrupting FSO communication. The disparity in a rain droplet leads to multiple reflections and refractions. The specific attenuation due to rain is $\alpha=a R b(\mathrm{~dB} / \mathrm{km})$, where $a, b$ are frequency dependent factors and $R$ is the rainfall rate $(\mathrm{mm} / \mathrm{h})[10]$. Rain drops have the size exceeding the wavelength of light signal, thus non-selective scattering predominates, but Mie scattering is still utilized to find the attenuation values. The expression for rain attenuation is: $\alpha_{\text {specific }}=1.076 R^{0.67}(\mathrm{~dB} / \mathrm{km})$.

The transmission of optical signal is also disrupted by attenuation caused by $\mathrm{CO}_{2}$ and other gases present in the atmosphere. The attenuation value is given by $\mathrm{Kim}$ and Kruse:

$$
\sigma=\frac{3.91}{V}\left(\frac{\lambda}{550 \mathrm{~nm}}\right)^{-q}
$$

where $V$ is the visibility $(\mathrm{km}), \lambda$ is the wavelength $(\mathrm{nm})$ and $q$ is the size of distribution of scattering particles (nm). The attenuation in Eq. 3 is the function of visibility only, which determines the defined particle size [11]. The values to be used in the above equation are given in Table 1 .

Table 1

Values to be used in Eq. 3

\begin{tabular}{|c|c|c|}
\hline$V[\mathrm{~km}]$ & $q[\mathrm{~nm}]$ & Visibility type \\
\hline \hline Over 50 & 1.6 & High \\
\hline $6 \ldots 50$ & 1.3 & Average \\
\hline $1 \ldots 6$ & $0.16 V+0.34$ & Haze \\
\hline $0.5 \ldots 1$ & $V-0.5$ & Mist \\
\hline Below 0.5 & 0 & Fog \\
\hline
\end{tabular}




\subsection{Proposed System}

The proposed system uses three components: transmitter, transmission medium and receiver. The transmitter has a pseudo-random bit sequence generator, continuous wave laser, NRZ pulse generator and Mach-Zehnder modulator. The medium is air using the FSO channel, and the receiver has a PIN detector and a Gaussian low pass filter. The relay nodes are placed in between the channel at the reception side, to enhance the system characteristics. The performance is measured using the BER analyzer and an optical power meter. The noise figure is maintained at $4 \mathrm{~dB}$ and the geometrical losses are considered. The system is analyzed by Optisystem 11.0 to have the lowest BER at $1550 \mathrm{~nm}$ for the $2.5 \mathrm{~Gb} / \mathrm{s}$ data rate.

Table 2

FSO link optimized parameters

\begin{tabular}{|c|c|}
\hline Characteristics & Values \\
\hline \hline Attenuation level & $\begin{array}{c}\text { Haze, } 2.37 \mathrm{~dB} / \mathrm{km} \\
\text { Light rain, } 3 \mathrm{~dB} / \mathrm{km} \\
\text { Medium rain, } 9 \mathrm{~dB} / \mathrm{km} \\
\text { Heavy rain, } 20 \mathrm{~dB} / \mathrm{km}\end{array}$ \\
\hline Amplifier gain & $30 \mathrm{~dB}$ \\
\hline Laser power & $30 \mathrm{dBm}$ \\
\hline Data rate & $2.5 \mathrm{~Gb} / \mathrm{s}$ \\
\hline Channel spacing & $0.8 \mathrm{~nm}$ \\
\hline Aperture size & $20 \mathrm{~cm}$ \\
\hline
\end{tabular}

The system is studied as a three-channel WDM system (the number of channels depends on the number of users, so it can be extended accordingly), optimized for different values of amplifier gain and power transmitted by taking into account the existing system, i.e. WDM with the use of optical amplifiers [7]. The beam divergence of the system is $2.5 \mathrm{mrad}$ and the remaining optimized parameters are shown in Table 2.

The system design is illustrated in Fig. 4. It employs an optical detector to receive the incoming optical signal, a directly modulated laser which is used as a pump to boost the signal level at a synchronized level which is based on its extinction ratio, and an EDFA with $5 \mathrm{~m}$ length. Serial relays are a far better choice than parallel ones, as the latter solution is not good for longer path links. The noise also adds up if synchronization is not performed properly [12].

\section{Simulation Results of WDM-FSO Amplifier System}

The system studied not only decreases complexity of the network but also that of its implementation. The simulations show the performance of the proposed system using relays. The range of more than $50 \mathrm{~km}$ is noticed for light rain, $60 \mathrm{~km}$ for the haze condition, up to $20 \mathrm{~km}$ for medium rain and to $8 \mathrm{~km}$ in heavy rain at the highest data rate of $2.5 \mathrm{~Gb} / \mathrm{s}$ as provided in Table 3 .

The results for heavy rain and haze from the BER analyzer are shown in Fig. 5. The eye diagram for haze is given in Fig. 6. The laser power is kept at $30 \mathrm{dBm}$, and the optical amplifier gain is set to an optimized value. The output of the BER analyzer shows that the eye opening is good in the relay-assisted system, thus assuring better SNR at the receiver.

Evaluation of the system shows that for light and medium rain conditions the link distance extends up to the orders of 50 and $2 \mathrm{~km}$ respectively, with the BER value of 6.1033 . $10^{-56}$ and $2.38721 \cdot 10^{-55}$ for acceptable transmission.

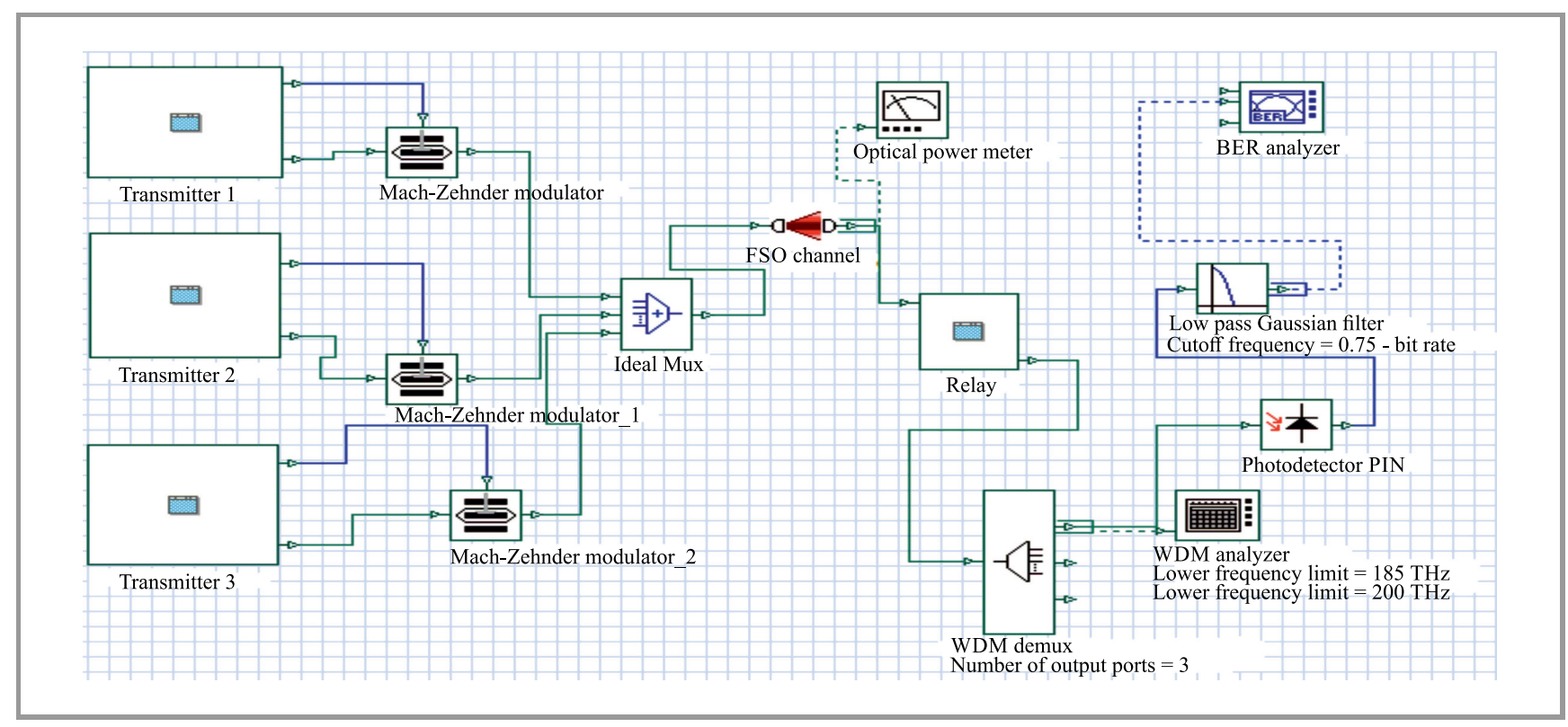

Fig. 4. Relay-assisted network. (See color pictures online at www.nit.eu/publications/journal-jtit) 
Table 3

Comparison of WDM-FSO amplifier-assisted and relay-assisted system

\begin{tabular}{|c|c|c|c|c|c|c|}
\hline \multirow{2}{*}{$\begin{array}{c}\text { Weather } \\
\text { condition }\end{array}$} & \multicolumn{3}{|c|}{ Amplifier-assisted WDM system } & \multicolumn{3}{c|}{ Relay-assisted WDM system } \\
\cline { 2 - 7 } & $\begin{array}{c}\text { Range } \\
{[\mathrm{km}]}\end{array}$ & $\begin{array}{c}\text { Minimum } \\
\text { BER }\end{array}$ & $\begin{array}{c}\mathrm{Q} \\
\text { factor }\end{array}$ & $\begin{array}{c}\text { Range } \\
{[\mathrm{km}]}\end{array}$ & $\begin{array}{c}\text { Minimum } \\
\text { BER }\end{array}$ & $\begin{array}{c}\mathrm{Q} \\
\text { factor }\end{array}$ \\
\hline \hline Light rain & 25 & $2.7534 \cdot 10^{-21}$ & 9.39 & 50 & $6.1033 \cdot 10^{-56}$ & 15.72 \\
\hline Medium rain & 9.3 & $2.70299 \cdot 10^{-20}$ & 9.14 & 20 & $2.38721 \cdot 10^{-55}$ & 15.62 \\
\hline Heavy rain & 4.5 & $3.51199 \cdot 10^{-20}$ & 9.11 & 8 & $6.2028 \cdot 10^{-56}$ & 15.71 \\
\hline Haze & 23 & $7.99541 \cdot 10^{-40}$ & 10.10 & 60 & $2.3769 \cdot 10^{-54}$ & 15.69 \\
\hline
\end{tabular}

(a)

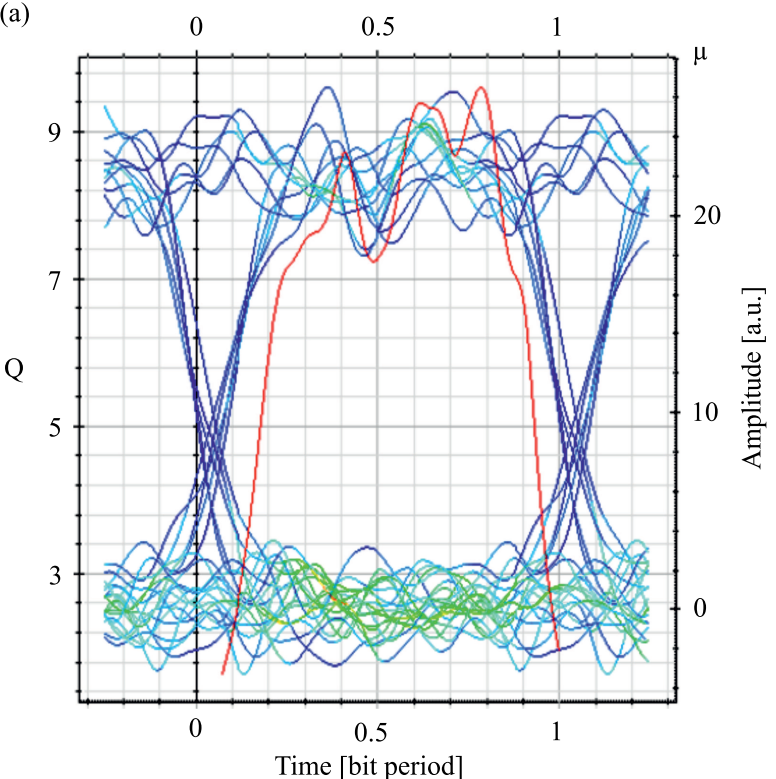

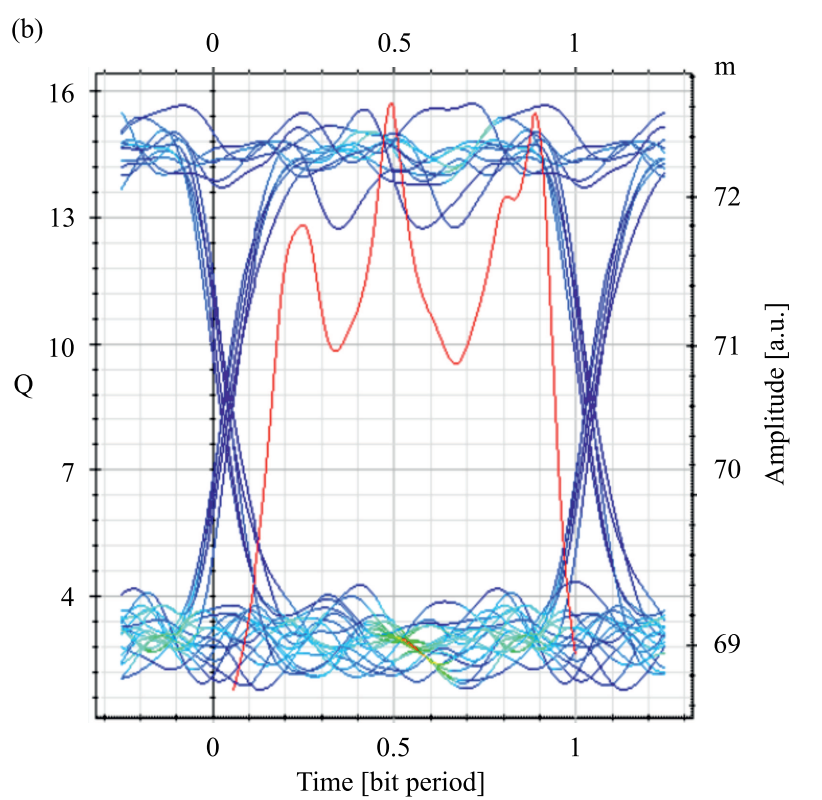

Fig. 5. BER output in heavy rain at $7 \mathrm{~km}$ using: (a) amplifier-assisted WDM system, (b) relay-assisted WDM system.
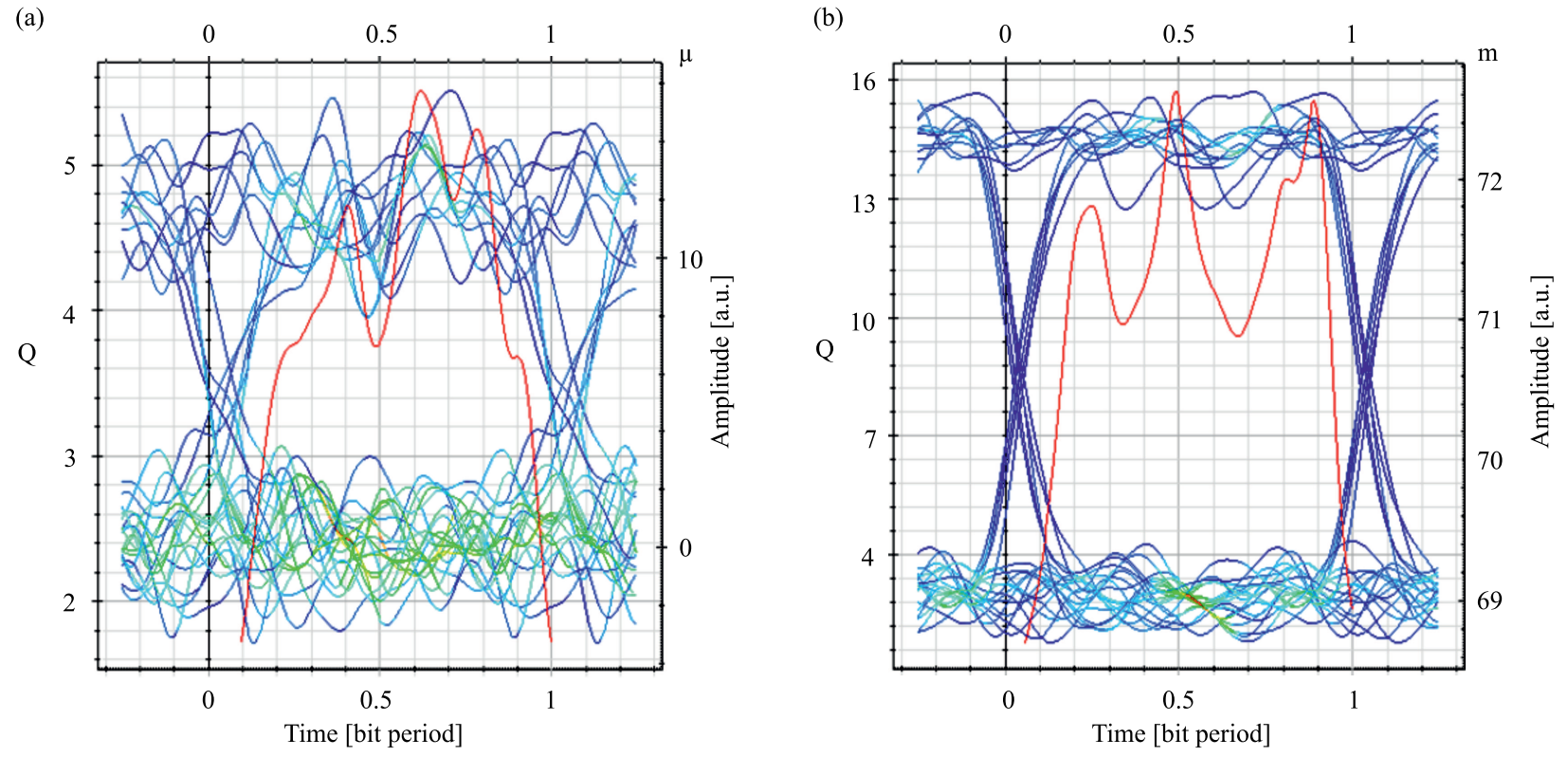

Fig. 6. BER output in haze condition at $60 \mathrm{~km}$ using: (a) amplifier-assisted WDM system, (b) relay-assisted WDM system. 
(a)

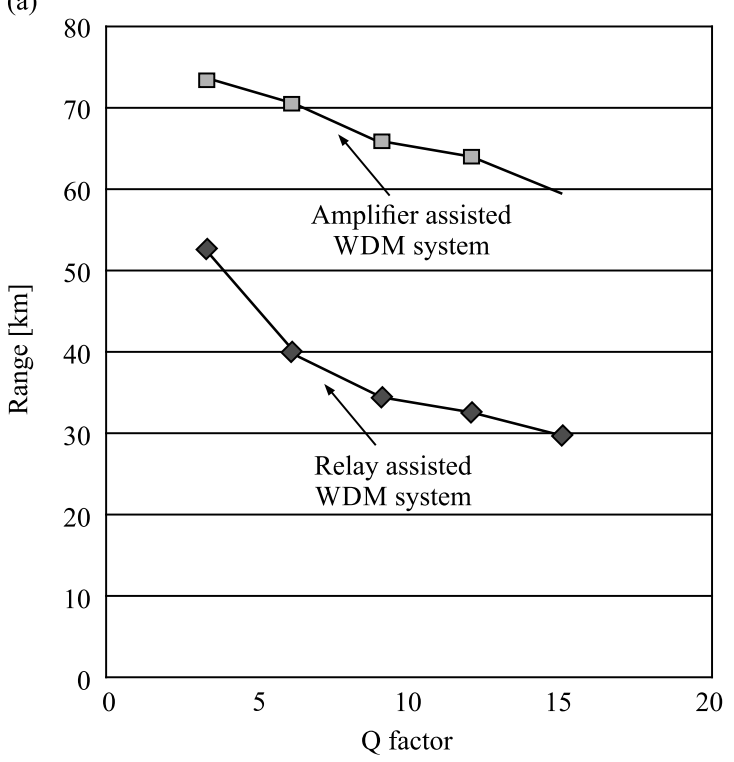

(b)

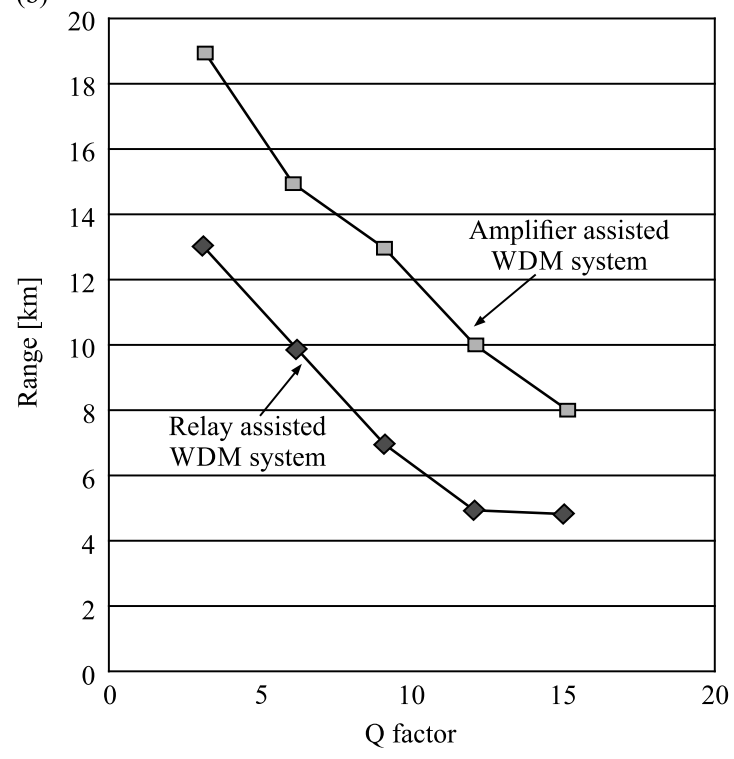

Fig. 7. Performance analysis of two systems in: (a) haze and (b) heavy rain scenarios.

For haze, the distance extends to $60 \mathrm{~km}$ having BER of $2.3769 \cdot 10^{-54}$, with performance remaining at an optimum level. Figure 7 describes the performance of the system's variation with the $\mathrm{Q}$ factor and the link range for an amplifier-assisted and a relay-assisted system. The graph shows an increasing rate of efficiency for the relayassisted system with acceptable SNR, and a good transmission range.

\subsection{Power optimization}

In the next step of the research procedure an effort was made to optimize the power level of the $\mathrm{CW}$ laser in order to achieve enhanced performance (Table 4). Originally, the

Table 4

Values observed within the system in various weather conditions at $2 \mathrm{dBm}$

\begin{tabular}{|c|c|c|c|c|}
\hline $\begin{array}{c}\text { Weather } \\
\text { condition }\end{array}$ & BER & $\begin{array}{c}\mathrm{Q} \\
\text { factor }\end{array}$ & $\begin{array}{c}\text { Link } \\
\text { range } \\
{[\mathrm{km}]}\end{array}$ & $\begin{array}{c}\text { Bit } \\
\text { rate } \\
{[\mathrm{Gb} / \mathrm{s}]}\end{array}$ \\
\hline \hline Haze & $2.6005 \cdot 10^{-6}$ & 15.66 & 54 & 2.5 \\
\hline Light rain & $5.7865 \cdot 10^{-54}$ & 14.67 & 50 & 2.5 \\
\hline $\begin{array}{c}\text { Medium } \\
\text { rain }\end{array}$ & $1.5634 \cdot 10^{-45}$ & 15.80 & 17 & 2.5 \\
\hline Heavy rain & $6.6754 \cdot 10^{-8}$ & 15.73 & 8 & 2.5 \\
\hline
\end{tabular}

transmitted power used for the system equaled $30 \mathrm{dBm}$, but after optimization the same system could offer better performance at a low power level, also when compared to the existing solutions. It has been observed that at lower power, performance of the WDM relay-assisted system is better, and that SNR achieved is optimum for an acceptable transmission.

\section{Conclusion}

In this article, a simulation of a WDM FSO serial relay assisted communication system is successfully performed. It has been observed that the performance of the system is affected, to a considerable degree, by haze and rain conditions. The results of the study show that the behavior of the system is outstanding in both types of weather conditions. For example, during haze and for the data rate of $2.5 \mathrm{~Gb} / \mathrm{s}$, the link range is $60 \mathrm{~km}$ with BER of $2.3769 \cdot 10^{-54}$ and an acceptable $Q$ factor. However, the study shows that the range of up to $50 \mathrm{~km}$ is achieved for light rain, $20 \mathrm{~km}$ for medium rain and $8 \mathrm{~km}$ for heavy rain. Finally, the power level has been optimized for the system, $2 \mathrm{dBm}$ is considered as a better solution here. At this level the performance achieved is better for any FSO system that is to be installed with its cost minimized.

\section{References}

[1] A. Malik and P. Singh, "Comparative analysis of point to point FSO system under clear and haze weather conditions", Wireless Person. Commun., vol. 78, no. 3, pp. 67-70.

[2] A. Malik and P. Singh, "Free space optics: current applications and future challenges", Int. J. of Optics, vol. 2015, Sep. 2015, pp. 1-7 (doi: 10.115/2015/945483).

[3] I. I. Kim, B. McArthur, and E. J. Korevaar, "Comparison of laser beam propagation at $785 \mathrm{~nm}$ and $1550 \mathrm{~nm}$ in fog and haze for optical wireless communications", in Proc. Information Technologies 2000, Boston, MA, USA, 2000, Optical Wireless Commun. III, vol. 4214 (doi: 10.1117/12.417512). 
[4] A. Hatziefremidis et al., "Bit error rate analysis along a slanted path link between UAVs and ground atations", in Proc. 15th Int. Conf. on Transpar. Optic. Networks ICTON, Cartagena, Andalucía, Spain, 2013, pp. 1-4 (doi: 10.1109/ICTON.2013.6602799).

[5] M. Gregory and S. Badri-Hoeher, "Characterization of maritime RF/FSO channel”, in Proc, 2011 Int. Conf. on Space Optic. Syst. and Appl. OCSOS, Santa, Monica, CA, USA, 2011, pp. 21-27.

[6] H. Maeda et al., "Performance degradation of long-distance optical WDM networks due to bandwidth limitations of wavelength multiplexers and de-multiplexers", IEEE Photon. Technol. Lett., vol. 11, no. 11, pp. 1509-1511, 1999.

[7] N. Dayal, P. Singh, and P. Kaur,"Performance enhancement in WDM-FSO system using optical amplifiers under different rain conditions", in Proc. Int. Conf. on Intell. Commun., Control and Devices ICICCD, Dehradun, Uttarakhand, 2015, pp. 293-298 (doi: 10.1117/12.417512).

[8] N. D. Chatzidiamantis et. al., "Protocols for relay-assisted free-space optical systems", in Proc. Glob. Telecomm. Conf. GLOBECOM 11, Houston, TX, USA, 2011, pp. 1-6 (arXiv: 1105.3835v1).

[9] J. Jeyarani and D. S. Kumar, "BER analysis of serial relay-assisted FSO systems over strong atmospheric turbulence", in Proc. 3rd Int. Conf. on Signal Process., Commun. and Network. ICSCN, Chennai, Tamil Nadu, India, 2015 (doi: 10.1109/ICSN.2015.7219865).

[10] F. Nadeem, E. Leitgeb, M. S. Awan, and S. Chessa, "Comparing lifetime of terrestrial wireless sensor network by hybrid FSO/RF and only RF", in 5th Int. Conf. on Wireless and Mobile Commun. ICWMC, Cannes, France, 2009 (doi: 10.1109/ICWMC.2009.29).

[11] H. Wu1 and M. Kavehrad, "Availability evaluation of ground to air hybrid FSO/RF links", Int. J. of Wireless Inform. Networks, vol. 14, no. 1, pp. 33-45, 2007.

[12] R. Boluda-Ruiz et al., "MISO relay-assisted FSO systems over gamma-gamma fading channels with pointing errors", Photon. Technol. letters, vol. 28, no. 3, pp. 229-232, 2015 (doi: 10.1109/LPT.2015.2492622).

[13] M. A. Kashani, M. Safari, and M. Uysal, "Optimal relay placement and diversity analysis of relay-assisted free-space optical communication systems", J. of Opt. Commun. Network, vol. 5, no. 1, pp. 37-47, 2013 (doi: 10.1364/JOCN.5000037).

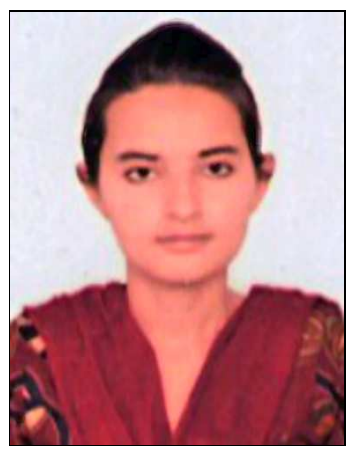

Navneet Dayal has received her M.E. in Electronics \& Communication Engineering from UIET, Panjab University, Chandigarh. Her research focuses on wireless optical communication systems.
E-mail: navidayal1992@gmail.com

University Institute of Engineering \& Technology

Department of Electronics \& Communication Engineering Panjab University

Chandigarh, India

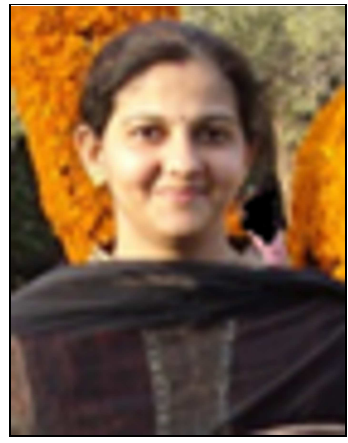

Preeti Singh is working as an Assistant Professor at the Electronics \& Communication Engineering Department in UIET, Panjab University, Chandigarh. She received her B.E. and M.E. degrees in Electronics and Communication Engineering. She obtained her Ph.D. degree in 2013. Her areas of interest include optical communication (wired and wireless), optical biosensors and cognitive neuroscience.

E-mail: preet_singh@pu.ac.in

University Institute of Engineering \& Technology

Department of Electronics \& Communication Engineering Panjab University

Chandigarh, India

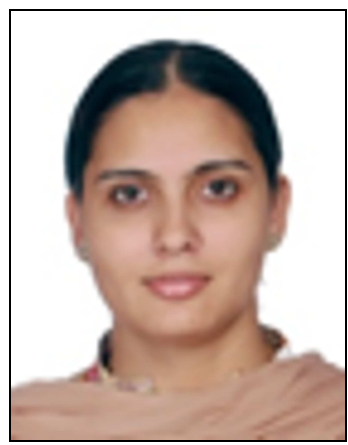

Pardeep Kaur is working as an Assistant Professor at the Electronics \& Communication Engineering Department in UIET, Panjab University, Chandigarh. She received her B.E. and M.E. degrees in Electronics and Communication Engineering. She is pursuing her Ph.D. in wireless sensor networks. Her areas of interest include optical communication and wireless communication. E-mail: pardeep.tur@gmail.com

University Institute of Engineering \& Technology

Department of Electronics \& Communication Engineering Panjab University

Chandigarh, India 